\author{
Piotr Nowak ${ }^{1 \star}$, Katarzyna Mastalska ${ }^{1}$ \\ and Jakub Loster ${ }^{2}$ \\ ${ }^{1}$ Laboratory of Parasitology, Department of \\ Microbiology, University Hospital in Krakow, 19 \\ Kopernika Street, 31-501 Krakow, Poland \\ ${ }^{2}$ Department of Infectious Diseases, University \\ Hospital in Krakow, 5 Sniadeckich Street, 31-531 \\ Krakow, Poland
}

Dates: Received: 01 December, 2015; Accepted: 29 December, 2015; Published: 30 December, 2015

*Corresponding author: Piotr Nowak, Laboratory of Parasitology, Department of Microbiology, University Hospital in Krakow, 19 Kopernika Street, 31- 501 Krakow, Poland, Tel: +4812/4247587; Fax: +4812/ 4247581; E-mail: parazytolog@gmail.com

www.peertechz.com

Keywords: Entamoeba histolytica; Entamoeba dispar; Entamoeba moshkovskii; Entamoeba histolytica sensu lato; Entamoeba histolytica sensu stricto; commensals of the large intestine; amoebiasis

\author{
Review Article
}

\section{Entamoeba Histolytica - Pathogenic Protozoan of the Large Intestine in Humans}

\section{Abbreviations}

E. histolytica: Entamoeba histolytica; E. coli: Entamoeba coli; E. nana: Endolimax nana; E. hartmanni: Entamoeba hartmanni; E. polecki: Entamoeba polecki; I. bütschlii: Iodamoeba bütschlii; ESR: Erythrocyte Sedimentation Rate; ALA Amoebic Liver Abscess; ALT: Alanine Aminotransferase; AST Aspartate Transaminase; COX-2; Cyclooxygenase-2; PGE 2 Prostaglandin E2; WHO: World Health Organization;

\section{Introduction}

Entamoeba histolytica (E. histolytica) is a unicellular organism of animal-parasitic infections, pathogenic protozoan from the family Entamoebidae. E. histolytica has identified and first described in literature by the doctor from St. Petersburg F. Aleksandrovich Lösch in 1875 [1]. Entamoeba histolytica cause dangerous syndrome called amoebiasis (amoebosis). At this point it should be noted that the term amoebosis is reserved only for the disease caused by Entamoeba histolytica; that term cannot be used in cases of the infections of other amoebas species of the family Entamoebidae.

Amoebiasis in humans may take the intestinal or parenteral form. Intestinal amoebiasis (the most common) is manifested by increased diarrhea, abdominal pain, flatulence, gas and increased body temperature. In contrast, in situations when the amoeba enters through the blood from the intestines to other organs of the body, where it can form abscesses (such as the liver, lungs, brain), we are talking about parenteral amoebiasis.

Untreated amoebiasis (especially intestinal infestations) can result in even sloping death. World Health Organization (WHO) estimates that each year around the world close to 500 million people get sick, and 100 thousand people die because of amoebosis-induced infection dysentery [2].

E. histolytica belongs to the cosmopolitan parasites, occurring throughout the globe. Particularly exposed to this parasite are people living and traveling to the tropical and subtropical zones (Asia, Africa, India, Indonesia, Mexico, South America, South Africa). These zones climatic conditions are optimal for the Protozoan cysts, which described the external environment can survive for many days. This fact contributes to the increase in the number of infections slider Entamoeba histolytica amongst people in tropical zones. According to Weinke to high-risk groups who are particularly vulnerable to being infected by E. histolytica include men with a homosexual orientation [3]. Prevalence, it means the incidence of infection is low in Poland and is about $1 \%$, while in tropical countries can claim up to $50 \%$. Most of the amoebiasis cases reported in Poland applies to people returning from a different climate zone and foreigners.

The digestive tract may be inhabited by several species of nonpathogenic amoebas in the family Entamoebidae, which include Entamoeba coli, Entamoeba hartmanni, Entamoeba polecki, Endolimax nana, Iodamoeba bütschlii [4-6]. The aforementioned amoebas are so called commensals of colon, which may settle the intestinal mucosa physiologically not attracting damages. There are only isolated reports in the literature that some commensals may lead to pathogenic actions in the human large intestine. There have been a couple of cases of diarrhea problems caused by infections of Endolimax nana, Entamoeba polecki and I. bütschlii in immunocompromised patients [6].

In 1925, Brumpt suggested that within the family Entamoebidae there are species Entamoeba dispar, which is morphologically 
identical with the pathogenic species Entamoeba histolytica [7]. This fact is confirmed in subsequent studies by analyzing the genetic material of both protozoa, which turned out to be different [8-10]. The species Entamoeba dispar is nonpathogenic, and now it belongs to the commensals of the colon. It was found that Entamoeba dispar occurs together with Entamoeba histolytica in the same areas in the world [5].

Ali, Haque and Tanyuksel have reported that within the family Entamoebidae there is one species of amoeba, which is morphologically identical with Entamoeba histolytica and is so called Entamoeba moshkovskii. This species, like the Entamoeba dispar, is nonpathogenic for the man and also belongs to the commensals of the colon. Entamoeba moshkovskii is not cosmopolitan, the literature mentions that it is endemic in Bangladesh, North America and South Africa $[6,11,12]$.

Nonpathogenic commensals of the colon should be necessarily vary with the species of pathogenic Entamoeba histolytica. This is of great importance from the point of view of pharmacotherapy, because the commensals of the colon usually do not require treatment.

\section{The purpose and objectives of the study}

In this work, efforts were made to present and systematize the most up-to-date information about the biology and pathogenicity of E. histolytica infection and symptoms of amoebiasis. However, special attention has been given to the broader subject of laboratory diagnosis of amoebiasis caused by Entamoeba histolytica. Discusses the usefulness, usability, and to reduce a number of analytical methods used in the diagnosis of various clinically as amoebiasis.

\section{Biology and morphology of E. histolytica}

Entamoeba histolytica can be found in the human body in the form such as infective cyst and vegetative form trophozoite.

Trophozoite: Trophozoite entity with a diameter equal to the 12 $60 \mu \mathrm{m}$, is surrounded by a three-tiered, lipid-protein cell membrane and creates characteristic ameboid pseudopodia that allow him to move and participate in phagocytosis, that is in the process of absorption of food particles. The cytoplasm is differentiated to ectoplasm and fine-grained endoplasm, which consists of cytosol and placed in him numerous cell organelles such as endosomes, lysosomes, Golgi apparatus, vacuoles with red blood cells and glycogen mass $[2,4,5,13,14]$. Trophozoite of Entamoeba histolytica does not contain mitochondria, which is why the energy distribution anaerobic wins the protozoans of glucose, which releases the stored glycogen mass $[15,16]$. A detailed process of all the metabolic pathways in the organism Entamoeba histolytica presented Loftus in the journal Nature [17].
Trophozoite of E. histolytica contains one round nucleus, in which the genetic material (DNA) concentrated in the form of a small, dense, centrally located karyosome and peripherally, evenly deployed chromatin. Presented to the construction of the cell nucleus is characteristic only of the species Entamoeba histolytica, as it has been shown that the protozoan in the family Entamoebidae is characterized by a high polymorphism of nuclei. The shape and position of the karyosome and the placement of chromatin in the cell nucleus is characteristic for each consecutive amoebas, which is used in their differential diagnosis. The structure of the nuclei of the amoebas is shown in Table 1.

Trophozoite is a form of this autonomic parasite, that is to say capable to perform all vital, including parasitic life in the body of the host. Trophozoite secretes specific proteolytic enzymes (e.g. hyaluronidase, cysteine proteinase) leading to degradation and cytolysis cells of tissues that have been attacked by E. histolytica [18]. The image of the trophozoite is shown in Figure 1 [19].

The cyst: The cyst of Entamoeba histolytica is a so called infective stage of parasite. The cyst is a trophozoite surrounded by specific membrane. The cyst is capable to survive in adverse conditions in the external environment for many days. This is due to the fact that the cyst is enclosed by multi-layer membrane containing, inter alia, chitin, which largely prevents the exchange of different substances between the interior of the cyst and the external environment [2]. The cyst with a diameter equal to the $10-20 \mu \mathrm{m}$ is usually round, contains 1, 2, 3 or 4 nuclei with karyosome and as trophozoite peripherally placed chromatin. The character mature cysts of $E$. histolytica contains glycogen focused within the cytoplasm in the form of irregular, spills, stains under the influence of Lugol's iodine in the form of a dark, orange-brown stains. It is very characteristic to the species Entamoeba histolytica. Glycogen mass occurs only at the stage of young cysts; mature cysts never contains it. In addition, the cysts are characteristic of the species Entamoeba histolytica. On the other side, cysts contains blunt finished chromatoidal bars. These structures are clearly visible in preparations stained with iodine or Trichrome (Gömöri-Wheatley technique) [4,5,13,14]. The form of cyst Entamoeba histolytica is shown in Figure 2 [19].

\section{Infection}

The main reservoir of the parasite in the environment is sick man who expel with feces protozoan cysts and becomes at the same time, the source of infection for other people [4]. The host becomes infected after oral ingestion of the protozoan cysts with the infected water or food (the so called fecal-oral transmitted way) [14]. Cysts Entamoeba histolytica are resistant to low $\mathrm{pH}$ of gastric juice, therefore are not destroyed in the light of the stomach. They are also resistant to

\begin{tabular}{|c|c|c|c|c|c|}
\hline Amoeba & $\begin{array}{l}\text { E. histolytical } \\
\text { E. disparl } \\
\text { E. moshkovskii }\end{array}$ & E. coli & E. hartmanni & E. polecki & E. nana \\
\hline $\begin{array}{l}\text { Peripheral } \\
\text { chromatin }\end{array}$ & $\begin{array}{l}\text { Fine granules, uniform in } \\
\text { size, beaded appearance }\end{array}$ & $\begin{array}{l}\text { Chromatin clumped and arranged } \\
\text { on the membrane, may appear as } \\
\text { solid dark ring }\end{array}$ & $\begin{array}{l}\text { Morphology similar to E. } \\
\text { histolytica sensu lato, chromatin } \\
\text { may appear as solid ring }\end{array}$ & $\begin{array}{l}\text { No peripheral chromatin or } \\
\text { chromatin clumped in large } \\
\text { granules on the membrane }\end{array}$ & $\begin{array}{l}\text { Usually no peripheral } \\
\text { chromatin }\end{array}$ \\
\hline
\end{tabular}




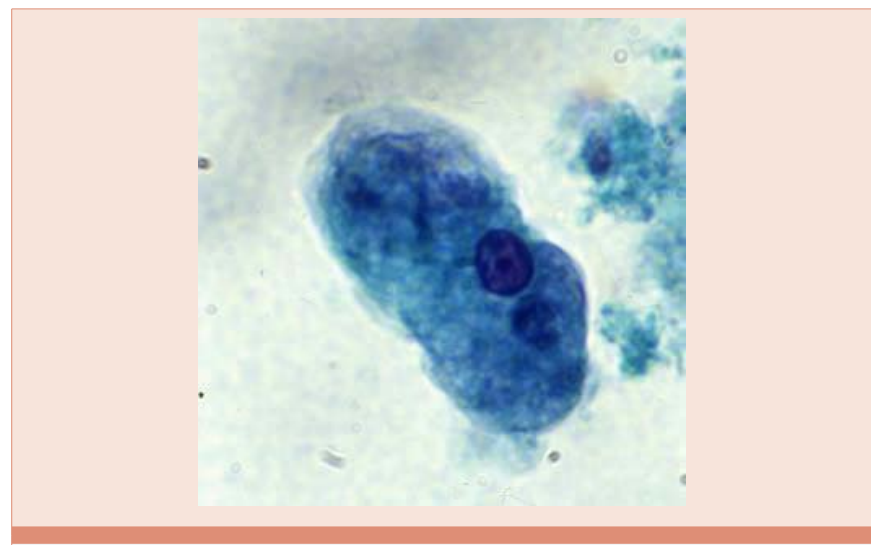

Figure 1: Trophozoite of E. histolytica (trichrome staining).

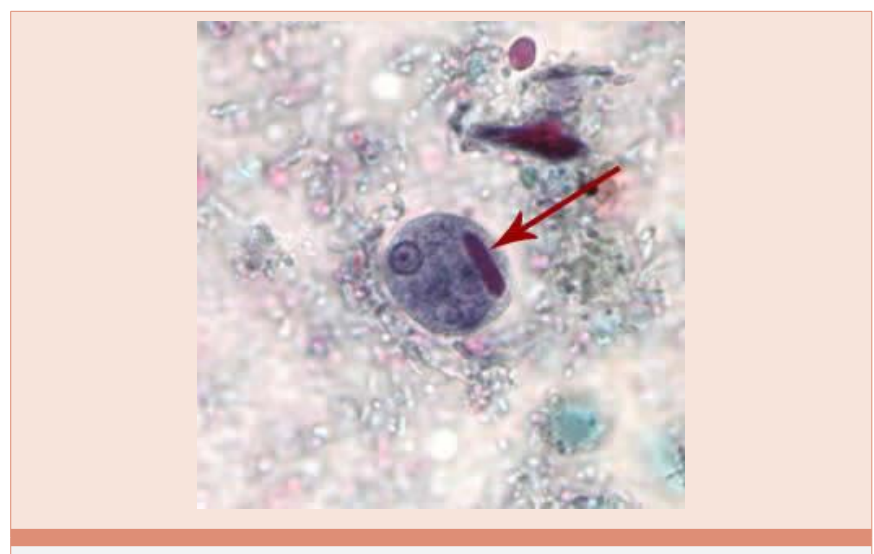

Figure 2: Cyst of E. histolytica (trichrome staining).

chlorination of water and various other chemicals, so they can easily survive in adverse environmental conditions, without losing its pathogenicity for the human host [2].

Contamination of food may occur with cysts as a result of the contact of food with the fly, which is a vector in the transmission of various cysts of protozoa, including Entamoeba histolytica and other pathogenic microorganisms (e.g. Salmonella, Shigella). In contrast to water cysts Entamoeba histolytica can get along with the feces of infected humans and animals through the release of wastewater into surface waters (rivers, lakes). Not without significance is also the placing of cesspools in the area of drinking water intakes. Predisposing factors to the infestation with E. histolytica are bad sanitary conditions, lack of running water in the house and breaking the basic rules of hygiene.

\section{The development cycle of E. histolytica}

This cycle was first described by Dobell in 1925 [6]. The cyst of Entamoeba histolytica after getting to the human body undergoes a transformation in the lower section of the small intestine in metacyst; this is called the excystation process. Trophozoite with four nuclei undergoes further multiple divisions, leading to the creation of very large mononuclear population capable of trophozoites invade the lining of the intestine, or attack other organs. Subsequently the trophozoites excrete food vacuole and other cellular organelle, resulting in take the characters of precysts. The life cycle of Entamoeba histolytica ends the process of encystation which resulted in precysts generating the areola in cyst forms. These cysts are then excreted in the feces $[2,13,6]$. Sick of amoebiasis man can excrete feces, even to tens of millions of cysts daily. Of course, with the feces are also excreted trophozoites of E. histolytica, but soon undergo degradation in terms of the external environment.

We do not know the incentives which the amoeba in the light of the intestine undergoes in the process of encystation and excystation. Eichinger and Stanley suggests that one of these factors can be adherence trophozoites to intestinal mucosa cells via receptor-specific lectin Gal/GalNAc [2,20].

\section{Pathophysiology and clinical signs of infestation}

Entamoeba histolytica makes a man so called amoebiasis (amoebosis). The most common clinical forms of amoebiasis are:

Acute dysentery, intestinal amoebiasis: The disease begins when trophozoites of E. histolytica are adhered to the epithelial cells of the colon with a special Gal/GalNAc lectin, a heterodimer comprised of three subunits with a total molecular weight $260 \mathrm{kDa}$ [21-22]. Stuck to the trophozoites mucosa secrete specific proteolytic enzymes such as hyaluronidase, cysteine proteinase, cathepsin $\mathrm{B}$, which produce a local inflammatory reaction, congestion, degradation attacked by amoeba cells and facilitate trophozoites further invasion of the intestinal submucosa tissue $[13,18,21,23]$. Trophozoites of amoeba cause induction of the enzyme cyclooxygenase-2 (COX-2) in the lining of the bowel, leading to an increase in the secretion of prostaglandin $\mathrm{E}_{2}\left(\mathrm{PGE}_{2}\right)$, which contributes to the stimulation of the inflammatory process [24]. Trophozoites of Entamoeba histolytica secretes specific polypeptides, the so-called amoebapores, which cause cytolysis cells of the lining of the intestine. This is due to the fact that the loss of cell organelles stimulate amoebapores in tissue affected by E. histolytica [25-27]. Cytolysis of cells is also caused by induction of the process of apoptosis by trophozoites Entamoeba histolytica; however, it is not thoroughly understood the mechanism of this process [28].

As far as the progression of the disease, as amended by the inflamed mucosa of the intestine are sores that develop abnormally and can rupture, leading to strong, massive hemorrhage which could even threaten the lives of those affected. Hence the name parasite (amoeba dysentery) and the name of the clinical form of the disease (amoebiasis). Acute intestinal amoebiasis is characterized by diarrhea with lots of mucus and blood in the stool, abdominal pain, nausea, bloating, elevated body temperature. Blood is considered elevated the amount of leukocytes, dating back to 15,000 in $\mathrm{mm}^{3}$ [4,5]. If the diarrhea is of great severity, lasts for a long time and is accompanied by her hemorrhages with ulcers, such person can claim the strong dehydration, electrolyte water management disorder, and in extreme cases, cardiovascular instability and collapse. Prolonged diarrhea is especially dangerous for infants and young children because of highly susceptible to dehydration and acid-base imbalance.

Chronic intestinal amoebiasis: Acute phase of amoebiasis, if it is not treated pharmacologically, most passes in the form of a chronic illness. This syndrome is characterized by alternating, bloodless diarrhea and constipation of varying severity symptoms of chronic 
ulcerative colitis, hypersensitivity of the intestines (colon irritable), an enlarged liver and soreness, low-grade fever, wasting and anemia $[4,5,13]$.

Amoebic hepatitis: The disease can develop as a consequence of acute intestinal amoebiasis as a result of a transfer by the blood of trophozoites Entamoeba histolytica from the intestine to the liver. Amoebic hepatitis manifests an enlarged liver soreness and raised the temperature of the body, chills and perspirations. Biochemical studies of blood appears to increase the activity of the enzymes ALT and AST [13].

Amoebic liver abscess (ALA): This is a very common and dangerous complication of acute intestinal amoebiasis. Trofozoity, that way the blood permeated from the intestine to the liver cause inflammatory changes, fibrosis and local necrosis of the liver lobules, resulting in the formation of an abscess, which is filled with a thick puss. In the amoebic abscess of the liver there are pains in the right upper quadrant, positive Chelmonski symptom, hepatomegaly, elevated body temperature, lack of appetite, weight loss. In addition, in the blood is considered leukocytosis and accelerated ESR $[2,13,29]$. If left untreated, amoebic liver abscess can be deadly.

Other forms of amoebiasis: In the wake of the amoebic liver abscess in the body of the person affected can create an amoebic abscesses in the various organs of the body, for example the lungs, pericardial, spleen, brain, kidneys or bladder. Abscesses may occur particularly often in people with AIDS [30-35]. Sometimes amoebic abscesses require surgical removal.

The invasion of subclinical dysentery: Some of the infections of Entamoeba histolytica can be carried out without any clinical signs (asymptomatic infestations). In this case, the protozoans, dreary life only in the light of the large intestine, without damaging its mucosa. Asymptomatic invasions of E. histolytica is recorded first and foremost on people living in temperate climates. However, recent scientific reports say that the invasions asymptomatic in most cases is the responsibility of the nonpathogenic species Entamoeba dispar or Entamoeba moshkovskii $[6,11,12]$.

\section{Laboratory diagnosis of amoebiasis}

Laboratory diagnosis of amoebiasis is quite difficult and consists of two main stages of the proceedings. In the first stage uses coproscopic methods, culture methods and serological methods [36-43].

At first it should be noted that coproscopic methods should be performed three times at intervals of 3 to 4 days, should be done before the drug therapy and then after its completion in order to assess the effectiveness of implementation of treatment. Unfortunately a lot of analytical laboratories performs only a single stool research towards intestinal parasites. This significantly reduces the sensitivity of the test and may lead to false negative test results.

Coproscopic methods in the laboratory are composed of direct preparations of feces $(0.9 \% \mathrm{NaCl}$, Lugol's iodine), thicken methods (flotation in a saturated solution of $\mathrm{ZnSO}_{4} \times 7 \mathrm{H}_{2} \mathrm{O}$ with centrifugation - the so-called Faust method, formol-ether method) and fecal smears stained with trichrome. In the preparations of these microscopically, using a 400x whole magnification, looking like cysts and trophozoites of Entamoeba histolytica [38,39,43]. In preparations of the saline stool direct you can find cysts, and trophozoites of amoeba, and the direct preparations with Lugol's iodine is looking for only protozoan cysts as headlining destroys trophozoites. In addition, trophozoites are usually present in diarrheal feces; in the formed stool he finds himself frequently only protozoan cysts. It should be added that the trophozoites of amoebas (including trophozoites Entamoeba histolytica) are volatile and bowel are quickly degraded in terms of the external environment. To detect trofozoity in feces, feces after his return as soon as possible should be tested in the laboratory. If it is not possible to carry out coproscopic methods, feces immediately after his return be should fix using Fixer PVA (polyvinyl alcohol), which creates a chance to trace the trophozoites in the material.

Cysts and trophozoites of Entamoeba histolytica microscopically differentiates with other, nonpathogenic amoebas of the digestive tract of a man of the family Entamoebidae such as E. coli, E. hartmanni, E. polecki, E. nana, I. bütschlii [4-6]. This step requires the laboratory diagnostic a wide knowledge of protozoology and large practical skills in the field of recognition of cysts and trophozoites above protozoa. Microscopically, it is estimated the shape and size of the cysts (cysts size measurement in preparations stained trichrome solution), the number of nuclei, the presence and shape of glycogen mass (preparations stained with Lugol's iodine) and the presence of other specialized organelles (e.g. the chromatoidal bars) [6]. However, the characteristics of the cysts Entamoeba histolytica and other cyst of amoebas in the family Entamoebidae is shown in Table 2.

As mentioned earlier, in differentiation of amoebas of the family Entamoebidae is useful knowledge of the construction of their nuclei. It has been shown that the shape and position of the karyosome and the placement of chromatin in the cell nucleus, is characteristic for each amoebas of the family Entamoebidae (Table 1). This fact is useful in differential diagnosis of amoebas in the family Entamoebidae.

Coproscopic studies are very useful in the diagnosis of intestinal amoebiasis, in which the person affected finds cyst and/or

\begin{tabular}{|c|c|c|c|c|c|}
\hline Amoeba & The shape of the cysts & $\begin{array}{l}\text { The size of the cysts } \\
{[\mu \mathrm{m}]}\end{array}$ & $\begin{array}{l}\text { The amount of } \\
\text { nuclei }\end{array}$ & Glycogen mass & Chromatoidal bars \\
\hline $\begin{array}{l}\text { E. histolytical } \\
\text { E. dispar/ E. moshkovskii }\end{array}$ & Round & $10-20$ & 1 to 4 & $\begin{array}{l}\text { Glycogen irregularly distributed } \\
\text { in the cytosol (mature cysts) }\end{array}$ & $\begin{array}{l}\text { + (large fingers, blunt } \\
\text { finished, always present) }\end{array}$ \\
\hline E. coli & Round to slightly oval & $10-33$ & $\begin{array}{l}\text { 1-18 } \\
\text { (usually 6-8) }\end{array}$ & $\begin{array}{l}+ \text { (1 large in } \\
\text { young cyst) }\end{array}$ & + (small, minor) \\
\hline E. hartmanni & Round & $4-8$ & $1-4$ & + & $+/-$ \\
\hline Endolimax nana & Round or oval & $5-14$ & 1-4 & $+/-$ & - \\
\hline
\end{tabular}


trophozoites of Entamoeba histolytica. According to Tanyuksel et al. in the diagnosis of intestinal amoebiasis microscopic methods has sensitivity $<60 \%$ and specificity in the range of $10-50 \%$ [6].

This is confirmed by Delialioglu, Evangelopoulos, Haque and other investigators stating that microscopic methods are characterized by low sensitivity and specificity for the diagnosis of amoebiasis $[37,38,40,43]$. Despite not very high values of sensitivity and specificity of microscopic methods, these methods are still very often used in the diagnosis of amoebiasis, because they are cheap and widely available in many laboratories around the world. Coproscopic methods are some kind of screening in the diagnosis of intestinal amoebiasis. However, finding the presence of cysts or trophozoites in stool, the diagnosis requires confirmation by other methods (e.g. molecular or serological tests), which reach higher values of sensitivity and specificity compared to microscopic methods. In addition to this (which will be mentioned later) cannot be distinguished microscopically species Entamoeba histolytica from nonpathogenic commensals as Entamoeba dispar or Entamoeba moshkovskii. Coproscopic methods are not suitable for diagnosis of hepatitis and amoebic liver abscess, where cysts and trophozoites in the feces of diseased person are rare. According to Tanyuksel et al. in the diagnosis of hepatic amoebic abscess test sensitivity of coproscopic methods is very low and reach $<10 \%$ [6].

In the laboratory diagnosis of amoebiasis are useful also culture methods, where material for culture may be feces or content such as abscess of the liver. Cultivation of Entamoeba histolytica may be carried out on the two-phase Robinson medium, constant Myjak's medium, or alternatively on a Diamond medium. With the obtained farms shall be then direct preparations and/or preparations stained with trichrome, which is assessed microscopically seeking cysts Entamoeba histolytica [6]. Currently the culture methods are used most often in the research-and-development labs to multiply amoebas (e.g. for further biochemical analysis).

In the diagnosis of amoebiasis are used widely all over the world serological methods to detect specific for Entamoeba histolytica coproantigens and antibodies. Serological methods include tests such as: IHA (Indirect Haemagglutination Assay), CIE (Counterimmunoelectrophoresis) and ELISA (Enzyme Linked Immunosorbent Assay). IHA and CIE method allows the detection of antibodies; in contrast, ELISA method allows detection of antibodies in serum and coproantigens in feces [6]. From all above mentioned methods listed here, currently most often used in the laboratories is ELISA method $[6,37,38]$ due to its simple methodology and not-soexorbitant price of diagnostic kits. In addition, ELISA method does not require costly, specialized laboratory equipment such as, for example, PCR methods; the result obtained in the form of a colour reaction be read visually, or by using a simple colorimetric ELISA reader.

In the diagnosis of amoebioasis the ELISA method can detect specific serum antibodies (immunoglobulin G (IgG)) against Gal/ GalNAc lectin of amoeba [6]. This test has the broadest application in the diagnosis of hepatic amoebic abscess (ALA); due to Tanyuksel his sensitivity is $>90 \%$ [6]. Due to the fact that the level of IgG antibodies may persist in the blood for a very long time, this test does not allow to determine exactly when there has been infection of E. histolytica. With the help of this test cannot be used to make a differential diagnosis between species E. histolytica, E. dispar and E. moshkovskii.

Using the ELISA method the laboratory can detect in the stool the specific for E. histolytica Gal/GalNac lectin coproantigens [6,3638,40,43-45]. According to Tanyuksel this test in the diagnosis of intestinal amoebiasis is characterized by very high sensitivity > $95 \%$, and a very high specificity also $>95 \%$, but just as coproscopic methods that test is completely not suitable for diagnosis of amoebic liver abscess [6]. In addition, TechLab, Blacksburg, Virginia offers the test Entamoeba histolytica II which is based on the ELISA method that allows the detection of specific for Entamoeba histolytica coproantigens; This test according to the manufacturer allows for differential diagnosis between species E. histolytica and E. dispar. Haque says that this test in the diagnosis of intestinal amoebiasis is characterized by sensitivity $>85 \%$ and specificity $>90 \%$ [40].

Laboratory diagnosis of E. histolytica cannot stop on the microscopy detection of cysts and/or trophozoites in the feces, as there are still two other protozoa, which are morphologically identical with Entamoeba histolytica. It is the already mentioned earlier species E. dispar and E. moshkovskii $[6,7,11,12]$. The species Entamoeba histolytica is necessary to diversify with the species E. dispar and/or $E$. moshkovskii, because of nonpathogenic amoebas do not require drug therapy.

For this reason, the second stage of the procedure includes diagnostic testing of genetic material (DNA) of amoeba, since this is the only way to distinguish the species Entamoeba histolytica from nonpathogenic commensals Entamoeba dispar or Entamoeba moshkovskii. The DNA of the above mentioned species you can vary the amoebas by using molecular biology methods such as: nested PCR, real-time PCR, LC-PCR, PCR-SHELA, Reverse Line Hybridization Assay [8-10,29,38,39,41,46-49]. These methods according to Tanyuksel et al., are characterized by high sensitivity (> 70\%) and a very high specificity (>90\%) in the diagnosis of intestinal amoebiasis, parenteral amoebiasis and amoebic liver abscess [6]. However, due to the very high cost and the need to have appropriate apparatus (e.g. thermal cycler), methods of molecular biology are used only in scientific centers and other highly specialized units. In Poland, an analysis of the genetic matherial of E. histolytica PCR carries out such as Interdepartmental Institute of Maritime and Tropical Medicine, Medical Academy of Gdansk. Differential diagnosis of Entamoeba histolytica/Entamoeba dispar is also possible through biochemical tests of amoebas by comparing the isoenzymes (hexokinase or phosfoglucomutase of amoebas) [47,50,51] or by ELISA test to detect the specific for Entamoeba histolytica or Entamoeba dispar coproantigens using specific monoclonal antibodies, for example, using the test TechLab Entamoeba histolytica II [40,45]. Detecting coproantigens in the feces by using ELISA test cannot differentiate species Entamoeba histolytica from Entamoeba moshkovskii, because there are still no commercial tests suitable for this purpose.

It should be add at this point that routinely performs a differential diagnosis between species of Entamoeba histolytica and Entamoeba dispar. This is due to the fact that the Entamoeba histolytica and Entamoeba dispar parasites are cosmopolitan, and Entamoeba 
moshkovskii parasite is not cosmopolitan. If you suspect that the test sample stool comes from the customers from the endemic presence of Entamoeba moshkovskii, differential diagnosis laboratory must extend it in the direction of the diagnosis of Entamoeba moshkovskii.

At the end of the consideration of the topic of laboratory diagnosis of amoebiasis we need to highlight the fact that where it is not possible to differentiate human pathogenic species of Entamoeba histolytica from nonpathogenic commensals Entamoeba dispar or Entamoeba moshkovskii (for example, by analyzing the genetic material) the parasite should be defined as Entamoeba histolytica sensu lato or E. histolytica complex. In addition, in 1997 the WHO and UNESCO announced that if the presence of amoeba dysentery is detecting only under microscopic examination, the result of the test should contain information that stated the presence of "Entamoeba histolytica/Entamoeba dispar" [40].

\section{Drug treatment of amoebiasis}

Pełzakowicy is currently used in the treatment of metronidazole, tinidazole, dehydremetin, chloroquine, and paromomycin $[5,14]$. The choice of drug, of course, depends on the type and severity of the amoebiasis, the presence or absence of organ abscesses and the age of the patient. The treatment of amoebiasis should be carried out by a specialist in infectious diseases. After treatment, it is necessary to perform a re-examination parasitological feces in order to assess the effectiveness of implemented drug therapy, because the literature reports of cases of resistance of E. histolytica for some medications.

\section{Prevention of transmission of amoebiasis}

A very important topic on amoebiasis is also prevention of Entamoeba histolytica infections, which consists of a few factors.

The first of these is parasitological examination of feces. These studies are mandatory in Poland for people returning from tropical and subtropical zones, where the amoeba can be delayed. In Poland the administrative supervision of persons returning from the tropics have Sanitary-Epidemiological Stations. It is very important to early detect infections with Entamoeba histolytica and quickly enter the appropriate drug therapy. This is important because that protects people from the environment of the patient before casual infection.

Parasitological stool examinations are also important to the people working in a variety of cafetery business, due to the transmission path, which have the nature of fecal-oral [14]. Early detection and treatment of vectors of cyst, eliminates the dysentery carriers in contact with food and thus protects against infection spreads.

Another important factor in the prevention of infections Entamoeba histolytica is boiling tap water for drinking. Cysts of amoeba dysentery are resistant to chlorination of water and other chemical agents, while in temperature of $\sim 100^{\circ} \mathrm{C}$ cysts die after a few seconds. Boiling water intended for drinking protects almost $100 \%$ from amoebiasis.

People travelling to the tropics should note also for the protection of food against flying insects, because the fly is a vector of cysts of pathogenic protozoans to humans, including cysts Entamoeba histolytica.

\section{Summary}

Modern medicine has many effective antiparasitic drugs used in treatment of amoebiasis. In addition, Stanley says that the research currently underway recombinant vaccine based on antigens Entamoeba histolytica, which is meant to protect against amoebiasis, including amoebic a liver-promising clinical data obtained on animal material [2]. Despite this, according to the WHO, 100 thousand people a year worldwide die due to amoebiasis and its complications $[2,52]$. Stanley points out that the mortality rate in the course of intestinal perforation tangled fulminant amoebiasis reaches $40 \%$ [2]. These figures confirm how dangerous is amoebiasis caused by Entamoeba histolytica. For this reason, modern medicine infectious diseases demands a thorough, sensitive and specific laboratory diagnosis of amoebiasis, allowing for quick and accurate confirmation of the diagnosed amoebiasis.

For a number of years in the laboratory diagnosis of amoebiasis was based on the finding in the stool with the coproscopic methods cysts and trophozoites Entamoeba histolytica. As this has been thoroughly presented in this article, in the light of current knowledge of such proceedings is insufficient. This is due to the fact that within the amoebas of the family Entamoebidae there are morphologically identical with Entamoeba histolytica species Entamoeba dispar or Entamoeba moshkovskii, which with the help of coproscopic methods cannot be distinguished from human pathogenic species of Entamoeba histolytica. In addition, the coproscopic method does not completely suitable for laboratory diagnosis of liver amoebic abscess and amoebic abscesses of other organs of a man. For this reason, modern laboratory diagnostics of amoebiasis should include serological methods for diagnosis of parenteral amoebiasis and methods of molecular biology (e.g. PCR), which, on the one hand, to achieve high sensitivity and specificity compared to the coproscopic methods and, on the other hand, allow you to distinguish the species of commensal Entamoeba dispar or Entamoeba moshkovskii from human pathogenic species Entamoeba histolytica.

\section{References}

1. Lübbert C, Wiegand J, Karlas T (2014) Therapy of Liver Abscesses. Viszeralmedizin 30: 334-341.

2. Stanley SL (2003) Amoebiasis. Lancet 361: 1025-1034.

3. Weinke T, Friedrich-Jänicke B, Hopp P, Janitschke K (1990) Prevalence and clinical importance of Entamoeba histolytica in two high-risk groups: travelers returning from the tropics and male homosexuals. J Inf Dis 161: 1029-1031.

4. Boczo? K, Dery?o A, Drewa G, et al. (2002) Parasitology and medical acaroentomology. PWN Warszawa 2002: 104-117.

5. Br?borowicz J, Brzezi?ska E, Cofta S, et al. (2004) Clinical Parasitology in a multidisciplinary approach. PZWL Warsaw 288: 337-339.

6. Tanyuksel M, Petri WA (2003) Laboratory diagnosis of amebiasis. Clin Microbiol Rev 16: 713-729.

7. Brumpt ME (1925) Étude sommaire de I '《e Entamoeba dispar >> n. sp. Amibe a kystes quadrinucléés, parasite de l'homme. Bull Acad Méd 94: 943952.

8. Hamzah Z, Petmitr S, Mungthin M, Leelayoova S, ChavalitshewinkoonPetmitr Pb (2006) Differential detection of Entamoeba histolytica, Entamoeba dispar, and Entamoeba moshkovskii to a single-round PCR Assy. J Clin Microbiol 44: 3196-3200. 
9. Qvarnstrom Y, James C, Xayavong M, Holloway BP, Visvesvara GS, et al. (2005) Comparison of real-time PCR protocols for differential laboratory diagnosis of amebiasis. J Clin Microbiol 43: 5491-5497.

10. Roy S, Kabir M, Mondal D, Ali IK, Petri WA Jr, et al. (2005) Real-Time PCR assay for diagnosis of Entamoeba histolytica infection. J Clin Microbiol 43: 2168-2172.d

11. Ali IK, Hossain MB, Roy S, Ayeh-Kumi PF, Petri WA Jr, et al. (2003) Entamoeba moshkovskii infections in children, Bangladesh. Emerg Infect Dis 9: $580-584$.

12. Haque R, Ali IKM, Clark CG, et al. (1998) A case report of Entamoeba moshkovskii infection in a Bangladeshi child. Int Parasitol 47: 201-202.

13. Dziubek Z, Janeczko J, Juszczyk J, et al. (2003) Parasites and parasitic diseases. Infectious and parasitic diseases. Dziubek Z. PZWL Warsaw 438444.

14. Virella G (2000) Microbiology and infectious diseases. Urban \& Partner Wroc?aw 415-418.

15. Clark CG, Roger AJ (1995) Direct evidence for secondary loss of mitochondria in Entamoeba histolytica. Proc Natl Acad Sci USA 92: 6518-6521.

16. Reeves RE (1984) Metabolism of Entamoeba histolytica Schaudinn, 1903 Adv Parasitol 23: 105-142.

17. Loftus B, Anderson I, Davies R, Alsmark UC, Samuelson J, et al. (2005) The genome of the protist parasite Entamoeba histolytica. Nature 433: 865-868.

18. Que X, Reed SL (2000) Cysteine proteinases and the pathogenesis of amebiasis. Clin Microbiol Rev 13: 196-206.

19. Upton SJ (-----) Animal parasitology. Entamoeba histolytica.

20. Eichinger D (2001) A role for a galactose lectin and its ligands during encystmnent of Entamoeba histolytica. J Eukaryot Microbiol 48: 17-21.

21. Dodson JM, Clark CG, Lockhart LA, Leo BM, Schroeder JW, et al. (1997) Comparison of adherence, cytotoxicity was, and Gal/GalNAc lectin gene structure in Entamoeba histolytica and Entamoeba dispar. Parasitol Int 46 225-235.

22. Mann BJ (2002) Structure and function of the Entamoeba histolytica Gal/ GalNAc lectin. Int Rev Cytol 216: 59-80.

23. Zhang Z, Le Y, Wang L, Seydel KB, Ellen Li, et al. (2000) Entamoeba histolytica cysteine proteinases with Interleukin-1 beta converting enzyme (ICE) activity cause ailments inflammation and tissue damage in amoebiasis. Mol Microbiol 37: 542-548.

24. Stenson WF, Zhang Z, Riehl T, Stanley SL Jr (2001) Amebic infection in the human colon induces cyclooxygenase-2. Infect Immun 69: 3382-3388.

25. Bruhn H, Riekens B, Berninghausen O, Leippe M (2003) Amoebapores and NK-lysin, members of the class of structurally distinct antimicrobial and cytolityc peptides from protozoa and mammals: a comparative functional analysis. Biochem J 375: 737-744.

26. Leippe M (1997) Amoebapores. Parasitol Today 13: 178-183.

27. López MC, León CM, Fonseca J, Reyes P, Moncada L, et al. (2015) Molecular Epidemiology of Entamoeba: First Description of Entamoeba moshkovskii in a Rural Area from Central Colombia. PLoS One 10: e0140302.

28. Ravdin JI, Croft BY, Guerrant RL (1980) Cytopathogenic mechanism of Entamoeba histolytica. J Exp Med 152: 377-390.

29. Khan U, Mirdha BR, Samantaray JC, Sharma MP (2006) Detection of Entamoeba histolytica using polymerase chain reaction in pus samples from amebic liver abscess. Indian J Gastroenterol 25: 55-57.

30. Campbell S (1993) Amebic brain abscess and menigocephalitis. Semin Neurol 13: 153-160.

31. Hung CC, Chen PJ, Hsieh SM, Wong JM, Fang CT, et al. (1999) Invasive amoebiasis: an emerging parasitic disease in patients infected with HIV in an area endemic for amoebic infection. AIDS 13: 2422-2428
32. Kubitschek KR, Peters J, Nickeson D, Musher DM (1985) Amoebiasis presenting as pleuropulmonary disease. West J Med 142: 203-207.

33. Lowther SA, Dworkin MS, Hanson DL (2000) Entamoeba histolytica/ Entamoeba dispar infection in human immunodeficiency virus-infected patients in the United States. Clin Infect Dis 30: 958-959.

34. Nath J, Ghosh SK, Singha B, Paul J (2015) Molecular Epidemiology of Amoebiasis: A Cross-Sectional Study among North East Indian Population. PLoS Negl Trop Dis 9: e0004225.

35. Reed SL, Wessel DW, Davis CE (1991) Entamoeba histolytica infection and AIDS. Am J Med 90: 269-271.

36. Abd Alla MD, Ravin JI (2002) Diagnosis of amoebic colitis by antigen-capture ELISA TEST in patients presenting with acute diarrhea in Cairo, Egypt. Trop Med Int Health 7: 365-370.

37. Delialioglu N, Aslan G, Sozen M, Babur C, Kanik A, et al. (2004) Detection of Entamoeba dispar/Entamoeba histolytica in stool specimens by using enzyme-linked immunosorbent assay. Mem Inst Oswaldo Cruz 99: 769-772.

38. Evangelopoulos A, Legakis N, Vakalis N (2001) Microscopy, PCR and ELISA applied to the epidemiology of amoebiasis in Greece. Parasitol Int 50: 185189.

39. González-Ruiz A, Haque R, Aguirre A, Castañón G, Hall A, et al. (1994) Value of microscopy in the diagnosis of dysentery associated with invasive Entamoeba histolytica. J Clin Pathol 47: 237-239.

40. Hawash YA, Dorgham LSh, Amir el-AM, Sharaf OF (2015) Prevalence of Intestinal Protozoa among Saudi Patients with Chronic Renal Failure: A Case-Control Study. J Trop Med 2015: 563478

41. Healy GR (1971) Laboratory diagnosis of amebiasis. Bull N Y Acad Med 47: 478-493.

42. Pillai DR, Keystone JS, Sheppard DC, MacLean JD, MacPherson DW, et al. (1999) Entamoeba histolytica and Entamoeba dispar: epidemiology and comparison of diagnostic methods in a setting of nonendemicity. Clin Infect Dis 29: $1315-1318$.

43. Tanyuksel M, Yilmaz H, Ulukanligil M, Araz E, Cicek M, et al. (2005) Comparison of two methods (microscopy and enzyme-linked immunosorbent assay) for the diagnosis of amebiasis. Exp Parasitol 110: 322-326.

44. Furrows SJ, Moody AH, Chiodini PL (2004) Comparison of PCR and antigen detection methods for diagnosis of Entamoeba histolytica infection. J Clin Pathol 57: 1264-1266.

45. Gonzalez-Ruiz A, Haque R, Rehman T, Aguirre A, Hall A, et al. (1994) Diagnosis of amebic dysentery to detection of Entamoeba histolytica fecal antigen by an invasive strain-specific, monoclonal antibody-based enzymelinked immunosorbent assay. J Clin Microbiol 32: 964-970.

46. Alver O, Topaç T, Töre O (2015) Evaluation of Two Methods (Nativ-Lugol Preparation and Enzyme-Linked Immunosorbent Assay) for Detection of Entamoeba histolytica in Stool Samples. Turkiye Parazitol Derg 39: 185-9.

47. Haque R, Ali IKM, Akther S, et al. (1998) Comparison of PCR, isoenzyme analysis, and antigen detection for diagnosis of Entamoeba histolytica infection. J Clin Microbiol 36: 449-452.

48. Verweij JJ, Laeijendecker D, Brienen EA, van Lieshout L, Polderman AM (2003) Detection and identification of Entamoeba species in stool samples by a reverse line hybridization assay. J Clin Microbiol 41: 5041-5045.

49. Verweij JJ, van Lieshout L, Blotkamp C, Brienen EA, van Duivenvoorden S, et al. (2000) Differentiation of Entamoeba histolytica and Entamoeba dispar using PCR-SHELA and comparison of antibody response. Arch Med Res 31: S44-S46.

50. Forsell J, Koskiniemi S, Hedberg I, Edebro H, Evengård B, et al. (2015) Evaluation of factors affecting real-time PCR performance for diagnosis of Entamoeba histolytica and Entamoeba dispar in clinical stool samples. J Med Microbiol 64: 1053-62. 
51. Martínez-García MC, Muñoz O, Garduño-Rodríguez G, Sánchez-Pares ME, Valadez-Salazar A, et al. (1990) Pathogenic and non-pathogenic zymodemes of Entamoeba histolytica in a rural area of Mexico. Concordance with serology. Arch Invest Med 21: 1457-152.

52. World Health Organization (1997) Amoebiasis. Wkly Epidemiol Rec 72: 97 99.

53. Gilchrist CA, Petri WA (1999) Virulence factors of Entamoeba histolytica. Curr Opin Microbiol 1999; 2: 433-437.
54. Haque R, Faruque AS, Hahn P, Lyerly DM, Petri WA Jr (1997) Entamoeba histolytica and Entamoeba dispar infection in children in Bangladesh. J Infect Dis 175: 734-736.

55. Haque R, Mondal D, Duggal P, Kabir M, Roy S, et al. (2006) Entamoeba histolytica infection in children and protection from subsequent amebiasis. Infect Immun 74: 904-909.

56. Rivera WL, Tachibana $\mathrm{H}, \mathrm{Kanbara} \mathrm{H}$ (1998) Fidel study on the distribution of Entamoeba histolytica and Entamoeba dispar in the northern Philippines as detected by polymerase chain reaction. Am J Trop Med Hyg 59: 916-921. 